A. Pramesh Rao, G. Swarup and Gopal-Krishna, eds.

\title{
Radio Recombination Lines from Starburst Galaxies: High and Low Density Ionized Gas
}

\author{
N.R. Mohan \\ Raman Research Institute, Bangalore 560080, India $\mathcal{G}$ \\ Indian Institute of Science, Bangalore 560012, India \\ K.R. Anantharamaiah \\ Raman Research Institute, Bangalore 560080, India \\ W.M. Goss \\ NRAO, Socorro, NM 87801, USA
}

\begin{abstract}
Radio recombination lines (RRL) at $8 \mathrm{GHz}$ and $15 \mathrm{GHz}$ detected from four starburst galaxies are shown to arise in compact high density HII regions, which are undetectable below $\sim 4 \mathrm{GHz}$. Detection of an RRL at $1.4 \mathrm{GHz}$ towards one galaxy and upper limits in the other three are consistent with the presence of an equal amount of low density diffuse gas. Continuum flux density measurements using the GMRT will be important in constraining the properties of the diffuse gas.
\end{abstract}

RRL and radio continuum studies of nuclear starbursts in galaxies are proving to be useful not only because of the absence of extinction but also because different density components of the ionized gas can be accessed through observations of RRLs at different frequencies (Zhao et al. 1996). We report a multi-frequency RRL and continuum study of four starburst galaxies.

We have observed RRLs at $1.4 \mathrm{GHz}, 8.3 \mathrm{GHz}$ and $15 \mathrm{GHz}$ using the VLA ${ }^{2}$ from four starburst galaxies : NGC 253, NGC 3628, and NGC 3690 and IC694 in the Arp 299 system. The two higher frequency lines were detected in all four galaxies and the line at $1.4 \mathrm{GHz}$ was detected only in NGC 253 . The line emission arises in the central $5-8^{\prime \prime}$ nuclear region in these galaxies. The peak line strengths for NGC 253 are about $5-8 \mathrm{mJy}$. The 8 and $15 \mathrm{GHz}$ lines are typically $0.5-1.0 \mathrm{mJy}$ in the other three galaxies and the $3 \sigma$ upper limits to the $1.4 \mathrm{GHz}$ lines are $\sim 1 \mathrm{mJy}$. We model the ionized gas as a collection of spherical uniform HII regions characterised by a single temperature, density and size. The total number of such HII regions is obtained by comparing the computed continuum and line emission for each model with the observed values. Other derived parameters are the flux of ionizing photons and the mass of the gas. Valid solutions are chosen based on constraints which are described in Anantharamaiah et al (1993). The models show that the $8.3 \mathrm{GHz}$ and $15 \mathrm{GHz}$

\footnotetext{
${ }^{2}$ The National Radio Astronomy Observatory is a facility of the National Science Foundation operated under cooperative agreement by Associated Universities, Inc.
} 

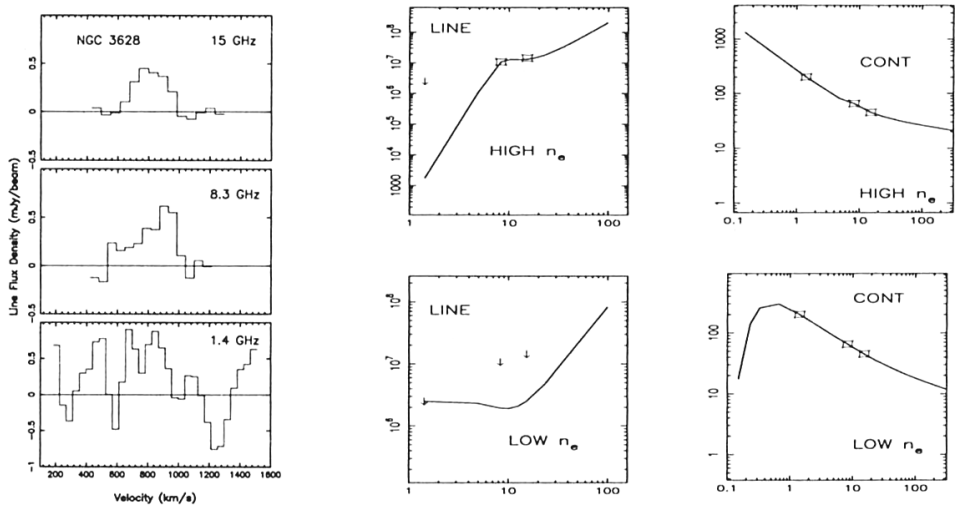

Figure 1. Left: The $2 \mathrm{~cm}, 3.6 \mathrm{~cm}$ and $20 \mathrm{~cm}(20 \mathrm{~cm}$ is a nondetection) RRL spectra towards NGC 3628. Right: The model predictions for the line and continuum flux densities for the high density $\left(\mathrm{T}_{e}=10000 \mathrm{~K}, \mathrm{n}_{e}=10^{4} \mathrm{~cm}^{-3}, \mathrm{l}=1 \mathrm{pc}\right)$ and low density $\left(\mathrm{T}_{e}=10000 \mathrm{~K}\right.$, $\mathrm{n}_{e}=100 \mathrm{~cm}^{-3}, \mathrm{l}=5 \mathrm{pc}$ ) gas versus frequency (in $\mathrm{GHz}$ ) in NGC 3628 . The vertical scales are in units of $\mathrm{mJy}-\mathrm{Hz}$ and $\mathrm{mJy}$ for the line and the continuum respectively. The results are similar for the other galaxies.

lines originate in a population of compact $(0.1-5 \mathrm{pc})$ high density (5000-50000 $\left.\mathrm{cm}^{-3}\right)$ HII regions with low total volume filling factor $\left(<10^{-4}\right)$. These lines arise from internal emission in the HII regions. Since the NIR and optical data imply much lower densities, this component is probably not detected in these bands due to high extinction. In all four galaxies, the photon flux neccesary for ionizing this gas is equal to or greater than that derived using conventional means. This result could lead to an upward revision of their star formation rates.

The above gas is practically undetectable at frequencies $\lesssim 4 \mathrm{GHz}$. Modeling the detected $\mathrm{H} 166 \alpha$ line at $1.4 \mathrm{GHz}$ from NGC 253 indicates that this line arises from low density $\left(10-100 \mathrm{~cm}^{-3}\right)$ diffuse $(5-100 \mathrm{pc})$ HII regions with an area filling factor $>0.1$. The upper limits to the $1.4 \mathrm{GHz}$ line for the other three galaxies give very similar results. Although the detection of lines from this component in the other three galaxies might be difficult with current instruments, measuring the continuum flux densities at $\nu<1 \mathrm{GHz}$ from the nuclear region using the GMRT will enable us to strongly constrain the properties of this gas.

These observations are a first step towards deriving properties of the ionized gas at different densities in starburst regions and hence towards studying the star formation at different time scales since the lifetime of an HII region depends on its density.

\section{References}

Anantharamaiah, K. R., Zhao, J. H., Goss, W. M., \& Viallefond, F. 1993, ApJ, 419,585

Zhao, J. H., Anantharamaiah, K. R., Goss, W. M., \& Viallefond, F. 1996, ApJ, 472,54 